Document downloaded from:

http://hdl.handle.net/10251/119579

This paper must be cited as:

Hadiwardoyo, SA.; Hernández-Orallo, E.; Tavares De Araujo Cesariny Calafate, CM.; Cano, J.; Manzoni, P. (2018). Experimental characterization of UAV-to-car communications.

Computer Networks. 136:105-118. https://doi.org/10.1016/j.comnet.2018.03.002

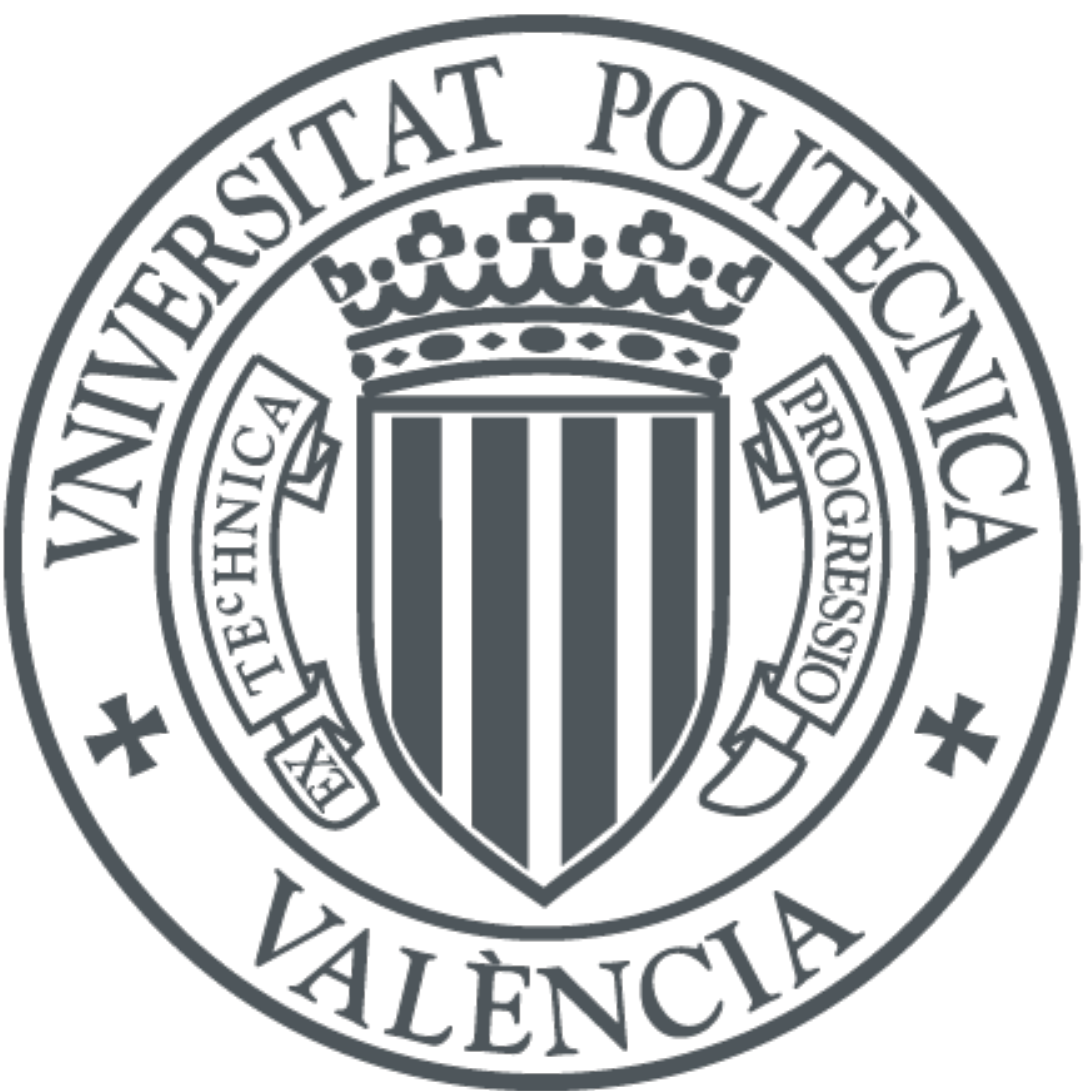

The final publication is available at

http://doi.org/10.1016/j.comnet.2018.03.002

Copyright Elsevier

Additional Information 


\title{
Experimental Characterization of UAV-to-Car Communications
}

\author{
Seilendria A. Hadiwardoyo*, Enrique Hernández-Orallo, Carlos T. Calafate, \\ Juan Carlos Cano, Pietro Manzoni \\ Department of Computer Engineering (DISCA), Universitat Politècnica de València, \\ Camino de Vera $s / n$, 46022 Valencia, Spain
}

\begin{abstract}
Unmanned Aerial Vehicles (UAVs), popularly known as drones, can be deployed in conjunction with a network of ground vehicles. In situations where no infrastructure is available, drones can be deployed as mobile infrastructure elements to offer all types of services. Examples of such services include safety in rural areas where, upon an emergency event, drones can be quickly deployed as information relays for distributing critical warning to vehicles. In this work, we analyze the communications performance on the link between cars and drones taking into account the altitude, the antenna orientation, and the relative distance. The presented results show that the communication between a drone and a car can reach up to three kilometers in a rural area, and achieves at least a fifty percent success ratio for the delivery rate at a 2.7 kilometer range. Finally, to allow integrating the communications link behaviour in different network simulators, the experimental results were also modeled with a modified Gaussian function that offers a suitable representation for this kind of communication.
\end{abstract}

Keywords: VANET, ITS, UAV, GRCBox, Real Implementation.

\footnotetext{
* Corresponding author

Email addresses: seiha@upv.es (Seilendria A. Hadiwardoyo), ehernandez@disca.upv.es (Enrique Hernández-Orallo), calafate@disca.upv.es (Carlos T. Calafate), jucano@disca.upv.es (Juan Carlos Cano), pmanzoni@disca.upv.es (Pietro Manzoni)
} 


\section{Introduction}

Intelligent Transportation Systems (ITS) are able to provide efficient solutions for traffic-related issues, such as safety and efficiency [1]. When attempting to make roads safer, ITS can provide systems that reduce the number of accidents taking place [2], along with safety-related applications [3].

Vehicle-to-Everything (V2X) paradigm that breaks down into the exchange of data between cars (V2V, or Vehicle-to-Vehicle), and infrastructure elements (V2I, or Vehicle-to-Infrastructure) which acts as relays towards a wider network or the Internet, is essential to provide ITS services and applications [4]. However, since vehicular networks are fast-moving and dynamic, the challenge comes when disseminating messages containing critical information that needs to be timely critical and as fast as possible for emergency and safety scenarios [5, 6]. Another challenge to consider in V2X communications is when the coverage area lacks infrastructure support. Although vehicular communications

15 can rely on various radio access technologies [7, e.g. using 4G LTE technology to support communications in areas with limited infrastructure like rural areas [8], major problems typically arise when the communications take place in areas that have no infrastructure support at all.

Unmanned Aerial Vehicles (UAVs) or drones (we will use these two terms interchangeably in this paper) are currently becoming an emerging solution for critical situations, i.e. disaster response like Search And Rescue (SAR) 9] and fire fighting [10]. In addition, compared to terrestrial communications, the adoption of UAVs not only offers a quick and flexible deployment, but also the chances of having Line-Of-Sight (LOS) with the receiver increases due to their higher altitude [11. Recently, thorough studies analyzed the capabilities of UAVs as communication agents, and their usefulness in several application scenarios [12, 13].

UAVs can also cooperate with ground vehicles in a particular network, allowing to improve the data exchanges between them. This approach offers benefits to multiple ITS applications [14, 15] like rescue and disaster assistance 
operations [16] and remote sensing [17]. In such cases, we typically rely on multiple UAVs to conform a network between themselves, creating what is known as a Flying Ad-Hoc NETwork (FANET). As a subclass of Vehicular Ad hoc NETworks (VANETs), FANETs differ from standard VANETs since they are characterized by highly mobile nodes moving freely in the 3D space; on the contrary, VANETs are restricted to 2D movements along streets. The challenges in FANET communications vary depending on the specific application. For instance, disaster monitoring introduces strong requirements such as low latency and very high information transmission rate (real-time video feed) [18. The use of multi-UAV systems can also be beneficial for improving the attainable transmission range and efficiency, as packets can be relayed and forwarded between UAVs to minimize the drawbacks of link interruptions [19].

One of the challenges of FANETs is obtaining an accurate radio propagation model, as this problem differs from typical scenarios addressed in the literature.

45 Most works focus on the link between UAVs and a static ground base [20, which typically has line-of-sight conditions. In another case [21, the authors modeled air-to-ground path losses with UAVs; however, the ground receiver was not a moving node. Instead, in this work, we focus on UAV-to-car communication, which is currently a very important topic [22, 23, 24, and that differs from the ground base case due to vehicular mobility. Thus, it becomes necessary to characterize the communications between UAVs and moving vehicles, and to derive a model that can be used in simulations combining FANET and VANET scenarios.

In this paper, we make a characterization of UAV-to-car communications based on real experiments aimed to foster the development of a communications model to be used in simulation studies. In our scenario, UAVs act as mobile RSUs (Road Side Units), enabling us to perform a study of vehicular communications between aerial and ground vehicles in the $5 \mathrm{GHz}$ band. The experiments were performed in a rural area of Valencia with actual field tests using vehicles and drones to determine the communications performance. In addition, based on the results obtained, we have modeled the packet delivery ratio in different 
scenarios (drone altitude, antenna rotation, and antenna orientation) using a modified Gaussian function.

The remainder of this paper is organized as follows: in the next section, we

65 provide an overview of related works regarding VANET scenarios that involve air-to-ground communications. In section 3 we describe the methodology, hardware, and software involved in our experiments. Then, in section 4 , we provide details about the scenario used in our experiments. Experimental results are presented and discussed in section 5 , followed by the modeling of our obtained

70 results in section 6. Finally, in section 8, we conclude the paper and refer to future works.

\section{Related Works}

UAVs have recently been adopted for a wide range of ITS solutions since they can become multi-purpose platforms for both rural and urban areas. Among their many applications, they can be used for surveillance [25], or become an aerial relay when the existing infrastructure fails to provide the desired services adequately, i.e., cell overload or outage [26]. In addition, they can be deployed for establishing a communications system when a disaster occurs [27]. This would allow an emergency communications system, essential for rescue operations, to be available. In post-disaster events, multiple UAVs can be also be easily deployed for search and rescue [28. In addition, multiple UAVs can conform a cooperative network, and define the best positioning strategy for better coordination in a distributed manner 29]. A typical application of such solutions includes homeland defense operations, where the network allows dynamic transitions from searching to the tracking of user agents and ground nodes [30. Other applications of deploying multiple UAVs (swarms) includes working in conjunction with Wireless Sensor Networks (WSNs) to monitor and detect potential disasters 31 .

The communication between UAVs and cars (air-to-ground communications) 90 is discussed in 22 . For instance, UAVs can assist vehicular networks by acting 
as intermediate relays. UAVs can also be deployed in an area where a disaster occurs, and therefore acts as bundle carriers and relays under the Delay-Tolerant Network (DTN) paradigm. In other work [23, the authors analyzed the possibility of deploying UAVs as flying base stations for communications to rescue vehicles in disaster events; in that scenario, vehicles should cooperate and maintain connections between them when terrestrial communication infrastructures are not available, a task that is assumed by UAVs. In such case, the authors in [32] analyzed the inter-connectivity of disconnected groups of cars that rely on UAVs. They studied the impact of increasing the number of cars in the group on the overall transmission quality. This case can also be applied in a scenario where a UAV is deployed as a Store-Carry-Forward node for vehicular networks [33. More efforts in this area include the deployment of VDNET (Vehicle-Drone hybrid vehicular ad hoc Network) [34, which offers better message transmission by equipping vehicle nodes with UAVs under its instruction so that it can communicate with other vehicle nodes over a greater transmission range. In another related work 24, networks of UAVs can be deployed throughout a city to achieve optimal information distribution. The presence of tall buildings and landmarks can cause disruption to the radio signal, resulting in frequent communication failures between vehicles. Hence, UAVs can assist on routing the information to the vehicles. In [35], authors considered the fusion of various ad-hoc networks, whether it is on air (FANET) or on the ground (VANET), to create a search and tracking-based guidance system.

In terms of the communications band, the authors of [36] proposed using an air-to-ground link at $5 \mathrm{GHz}$. The characterization was done using WiFi-enabled UAVs. In particular, WLAN operations using 802.11a is preferred for economic reasons. The authors in [37] have discussed the suitability of different frequency bands, showing that communication can suffer from severe interference if it operates in unlicensed Industrial, Scientific and Medical (ISM) bands due to their high usage. Hence, a licensed band such as the one adopted by IEEE 802.11p ${ }_{120}$ (5.9GHz band) is preferable to limit the effects of interference. To further emphasize on this problem, Fabra et al. 38] proved that FANET communications 
in the $2.4 \mathrm{GHz}$ band are prone to provide very poor performance, especially when the radio control operates in the same frequency band (majority of cases). When considering the cost, the power consumption, and the complexity, the authors in [39] relied instead on the IEEE 802.15.4 standard. They find that performance levels are inadequate when used as a mesh network, a basic requirement to deploy FANETs. Authors in [40], proposed a MAC protocol that assumes the use of directional antennas in FANET applications, as this introduces additional advantages in terms of transmission range compared to using a traditional omnidirectional antenna. However, in our work, we will study the general case of a $5 \mathrm{GHz}$ link using an omnidirectional antenna, as this is the most common situation in real scenarios.

Our work addresses the challenge of deploying a mobile infrastructure to support vehicular networks when there is an infrastructure shortage in a given target area. Notice that UAVs can offer line-of-sight communications for longer distances, which cannot always be achieved by car-to-car communications. So, we start by measuring the network performance for UAV-to-car communications in a rural area. In particular, we will assess the packet delivery ratio regarding data broadcasted from a flying UAV to a moving vehicle. Then, using the results obtained from such real experiments, we will create a model that allows estimating the packet delivery ratio based on distance. The architecture of the proposed network will be detailed in the following section.

\section{Architecture Overview}

In this section we start by providing a general overview of the envisioned scenario, and we then detail the proposed architecture, including the data flow and the different elements involved. Our proposed on board unit, named GRCBox [41, is also introduced at the end of this section.

\subsection{General Overview}

Our ultimate goal is to build an environment where UAVs can be deployed to assist communications in an area with limited or no infrastructure support. 


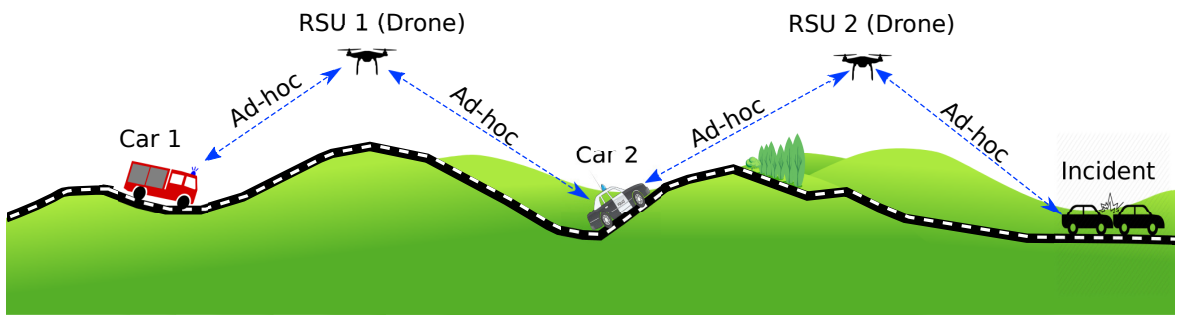

Figure 1: Drones as Mobile RSUs.

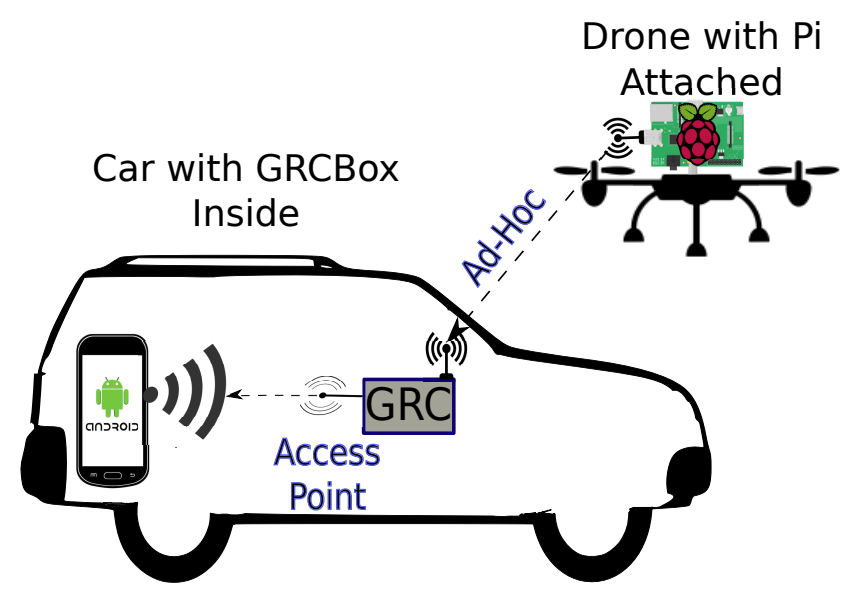

Figure 2: Flow of Packets from UAV (Drone) to Car.

Specifically, UAVs should be placed strategically to cope with those scenarios where vehicle-to-vehicle communications are hindered by the lack of line of sight. As depicted in Figure 1, the UAVs can be located near the top of surrounding hills causing line-of-sight obstructions between cars. Such UAV-assisted communications infrastructure would be useful when detecting a remote accident, especially if ground vehicular communications are not reliable due to large distances and non line-of-sight restrictions.

\subsection{UAV-to-Car Communications}

Our work focuses on measuring the effectiveness of point-to-point ad-hoc 160 communications between UAVs and cars, which can eventually conform the big picture detailed in the previous subsection. In particular, for this experimental 
work, appropriate hardware/software are required to measure the packet delivery ratio, the range, and to analyze the data obtained. Three different devices are used in our communications experiments: a Raspberry Pi mounted on the drone, an Android smartphone, and, finally, the GRCBox, which is a car onboard unit that is endowed with multiple communication interfaces, including ad-hoc communication capabilities. The GRCBox is placed on the car, along with the Android device that works as the end receiver.

Taking into account the widespread adoption of smartphones worldwide, this configuration can be a non-expensive and fast solution to deploy ITS equipment. For our experiments, we developed a test application for Android that was installed on the Android smartphone. This application allows the driver to receive data from the drone via the GRCBox. Regarding the drone itself, we embedded a Raspberry Pi on it. The former is used to generate packets periodically, broadcasting them via the ad-hoc network created ${ }^{1}$. In order to join the ad-hoc network, a car on-board unit (GRCBox) is needed, as the Android device cannot operate in this mode by default.

The packet flow generated is depicted in Figure2, Each packet is transmitted from the UAV to the car on-board unit (GRCBox) via an ad-hoc network. Since the GRCBox also provides an access point inside the vehicle, it acts as a packet relay, forwarding the packets received to the Android device connected to the GRCBox.

\subsection{GRCBox Overview}

The GRCBox is required for providing ad-hoc communications between car and drone, using the $5 \mathrm{GHz}$ band. In our experiments, we use an Android device to launch the application specifically developed for this purpose. Notice that Android devices do not offer ad-hoc connectivity by default, requiring the device to be rooted in order to achieve it, a requirement that is not practical for end users. Thus, our proposed GRCBox emerges as a solution to bypass

\footnotetext{
${ }^{1}$ Note that this device differs from the embedded UAV flight controller.
} 


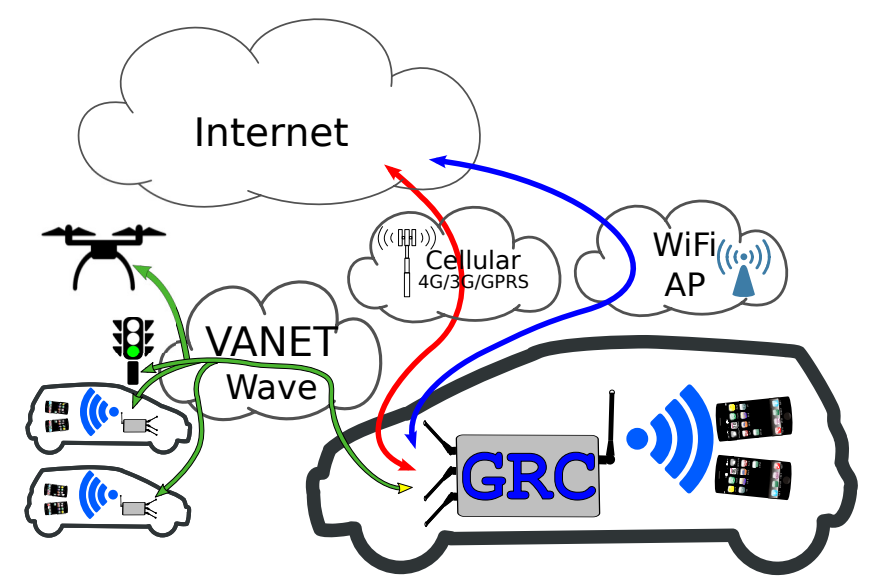

Figure 3: GRCBox Architecture For instance, one network interface can connect to a WiFi access point, and yet another interface can be used to connect to a $4 \mathrm{G}$ cellular base station. Figure 
3 shows a descriptive diagram of the GRCBox connectivity features.

Several services are provided by the GRCBox. GRCBox's inner interface acts as a soft-AP (Access Point) for smartphones. Once these smartphones are connected to the GRCBox, they can access the services that run on the external networks. Since every connection is forwarded by the GRCBox, any application needing to use an available interface that differs from the default ones (providing

Internet connectivity) must explicitly notify the GRCBox about it. These steps require rules that are defined by rule type, interface name, protocol, source port, source address, destination port, and destination address.

In our experiment, we will use the GRCBox as a receiver for packets that are broadcasted by the drone at the sender's side. The GRCBox is placed within the vehicle (see Figure 22. This GRCBox can act as the entry gate of packets that travel in the ad-hoc network connecting the drone and the car. The packets are then delivered to the passengers equipped with a smartphone running our GRCBox-aware application. Since in this experiment packets are broadcasted, GRCBox rules must be defined before the reception of packets in order to have a match in terms of associated ports and interfaces.

Regarding the drone, it is equipped with a Raspberry Pi configured to send packets in ad-hoc mode. This way, the Raspberry $\mathrm{Pi}$ on the drone and the GRCBox inside the car can transparently communicate through the same adhoc network. The Raspberry Pi's antenna in the drone has the same frequency band as the GRCBox's antenna (5 GHz).

\section{Experimental Settings}

In this section, we start by providing an overview of the location where experiments took place. Afterward, we detail the experimental tools used. Finally, we analyze the data gathered in our experiments.

235 4.1. Experimental environment

For the experimental tests, we have selected a rural area located on the outskirts of Casinos, a small town located to the west of Valencia, Spain. The road 


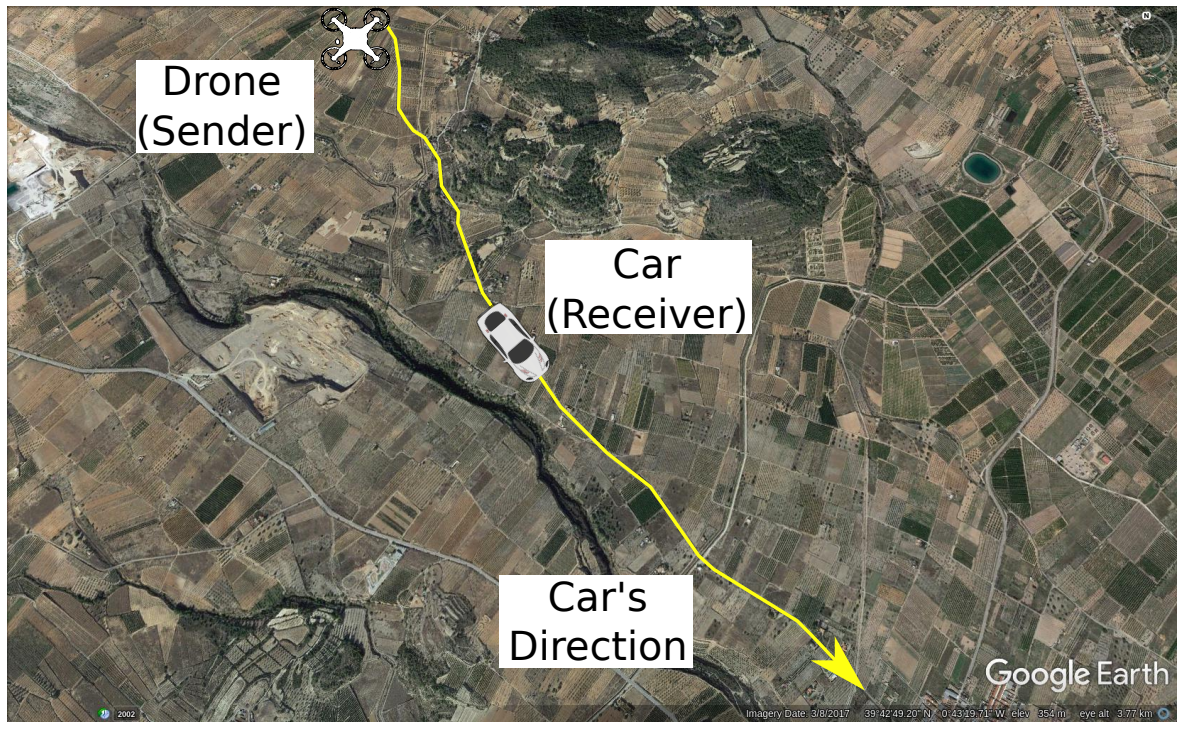

Figure 4: Satellite View of the target road near Casinos.

was located far from the urban area itself, and thus interference was minimal when operating in the $5 \mathrm{GHz}$ frequency band. As depicted in the aerial view (see Figure 4, we have selected a path for the vehicle that is more than 3 kilometers long.

The experiments were taken place in a Mediterranean climate on sunny summer season. At the time of the experiments, no precipitations were present. The wind speed was spotted to be very minimum, in which it was a typical weather condition in a valley surrounded by hills. Hence, the weather condition did not significantly affect the communication performance in the experiment.

In terms of the surface surrounding the experiment location, the trajectory experienced different elevation points (see Figure 5). From the starting point to the destination point, the road is found to be running downhill. Specifically, as detailed in Figure 6a, the elevation at the starting point is about 400 meters, and the final point is about 320 meters above the sea level. If we calculate the LOS (Line-of-sight) between the drone and the vehicle, we can see in Figure 6b, that depending on the drone's altitude, the worst case line-of-sight conditions are expected when the vehicle is located about 900 meters away from the 


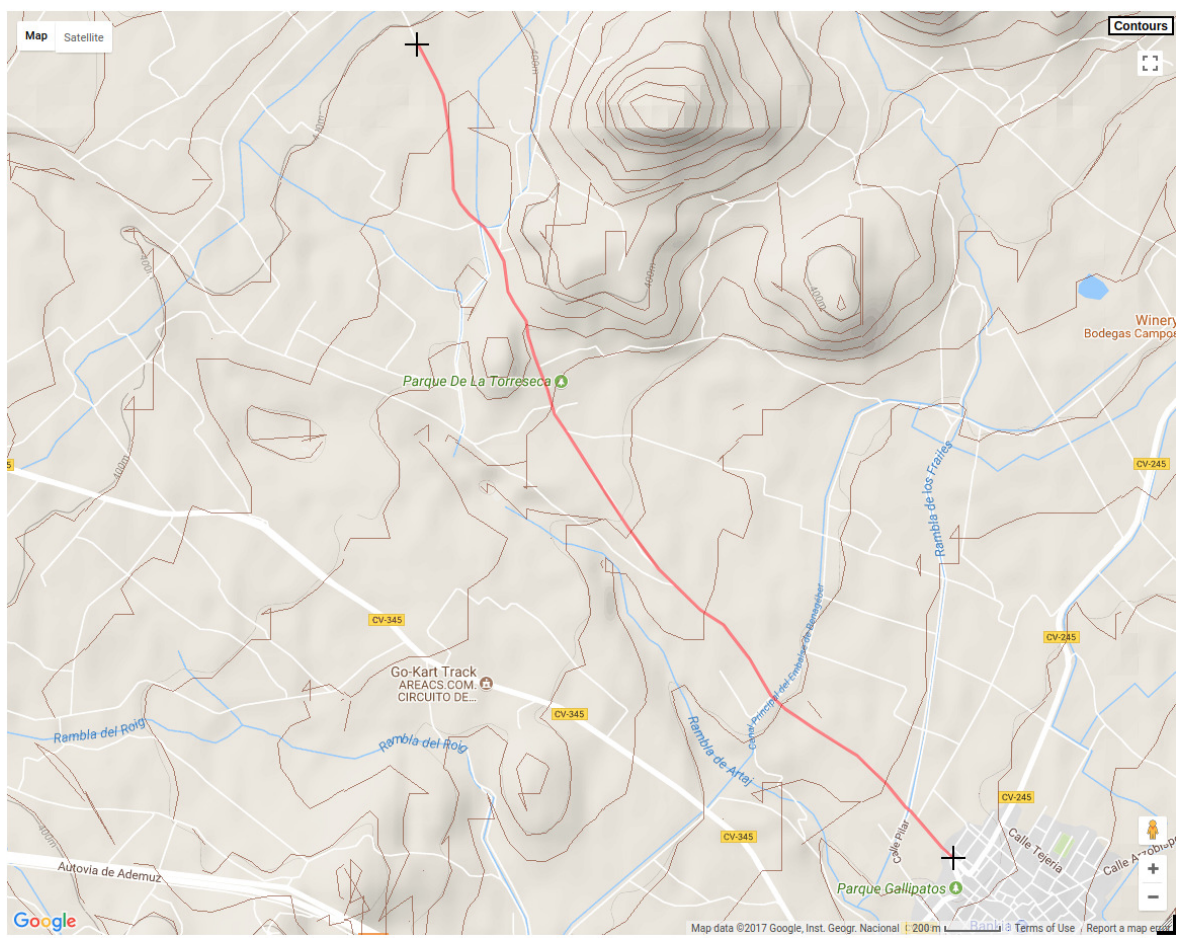

Figure 5: Topographic Map of Casinos. phone. This way, the car can seamlessly join the ad-hoc network. While moving 


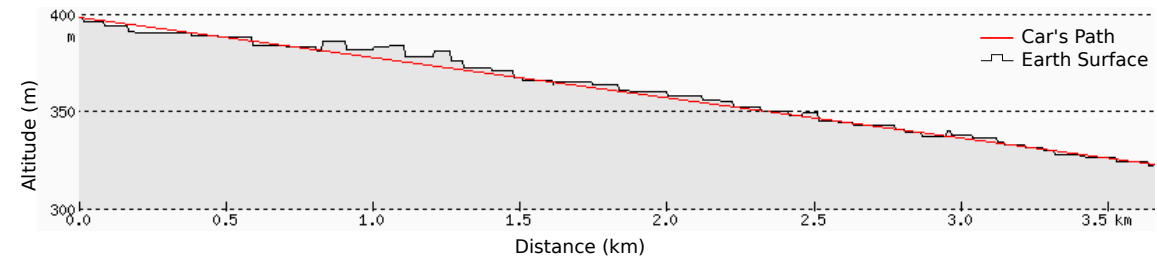

(a) Path followed by the ground vehicle.

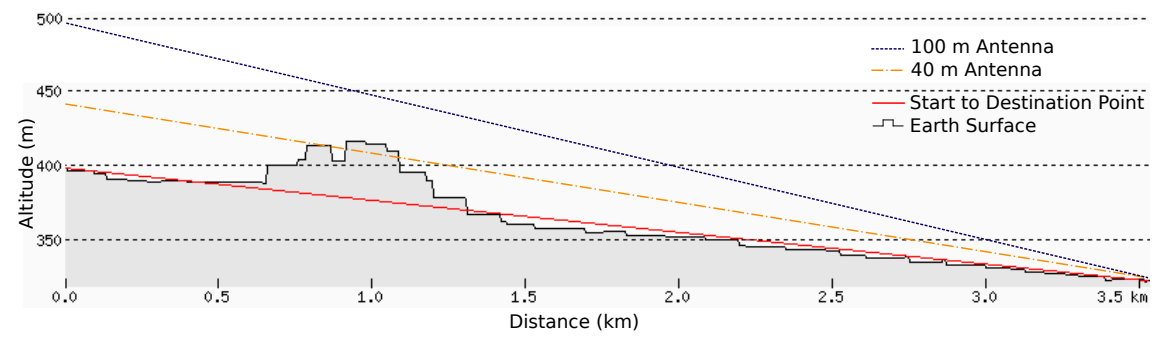

(b) LOS conditions experienced when the signal source is located at the Initial Starting Point for different drone altitudes.

Figure 6: Elevation profiles relevant to our experiments.

and receiving packets, the car will record its geographical location. This way, by continuously retrieving the geographic information, the receiver (in this case, the car) can determine with great accuracy the GPS coordinates associated to the reception of each particular packet.

At the sender side (Raspberry Pi embedded in the drone), we developed a Java application in order to generate broadcast messages. This test tool was tuned for a packet generation rate of $10 \mathrm{~Hz}$, each with a size of approximately 1.4 Kbytes, being each packet numbered with a sequential id. In addition, the timestamp and GPS location of the sender are also carried in the packet. These transmission parameters are similar to those of typical Decentralized Environmental Notification Messages (DENM) 42 in the context of vehicular networks. Also notice that, since all packet transmissions are broadcasted, the transmission rate is limited to $6 \mathrm{Mbps}$. The location information was obtained from the drone's GPS device by using the MAVLink protocol, that enables communication between the Raspberry Pi and the drone's hardware.

At the receiver's side, the packets were received by the Android smartphone 


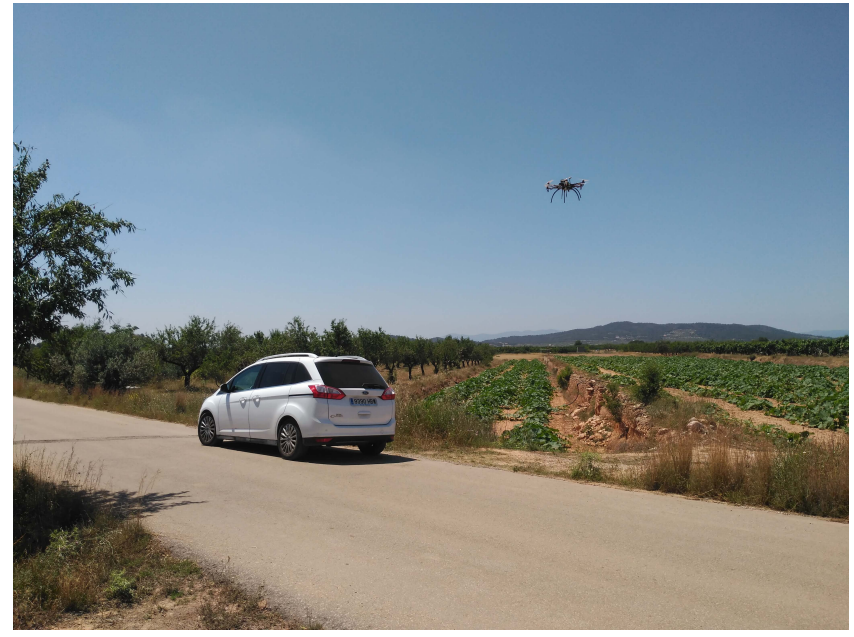

Figure 7: Real View of UAV-to-Car Communications in Casinos.

using the GRCbox as a relay. We have developed a specific Android-based application for this purpose. This application provides the rendering of information received from the drone. The tool is also used for measuring the packet delivery ratio in the test scenarios. It is relevant to point out that our Android-based application is fully compatible with GRCBox, meaning that the user does not need to configure the connection to the GRCBox interface. Instead, the application contains libraries and plugins that allow connecting to the GRCBox module in a seamless manner. Once the smartphone is connected to the access point of the GRCBox (inner interface), a bidirectional communications relay is established with the outer interface (ad-hoc network). In this application, packet reception is started when, at the receiver's side, the user presses the receive button. The packets received are then recorded and stored in a log file until the user stops the application. The stored variables are then measured to determine which have more impact on the communication's quality.

\subsection{Data Analysis}

The log file generated from the experiments contains all data required to measure the packet delivery ratio at different distances, including the geo- 


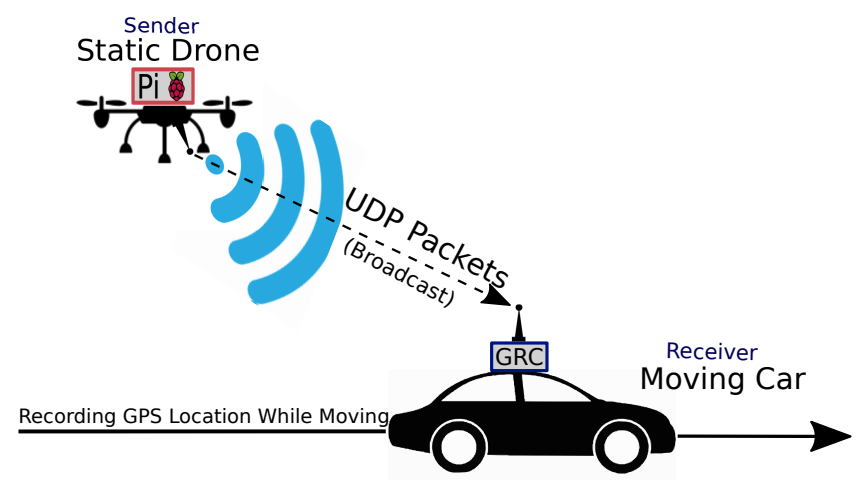

Figure 8: UAV-to-Car Communications in our Experiment's Scenario.

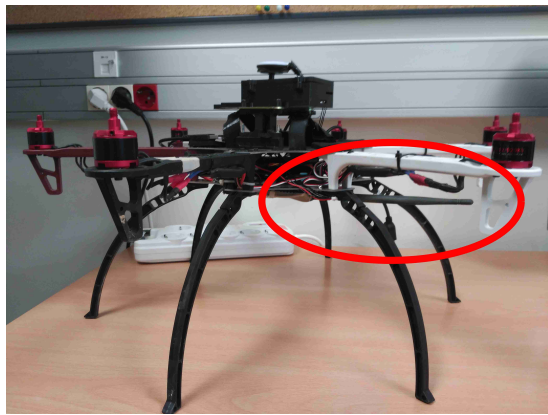

(a) Horizontal

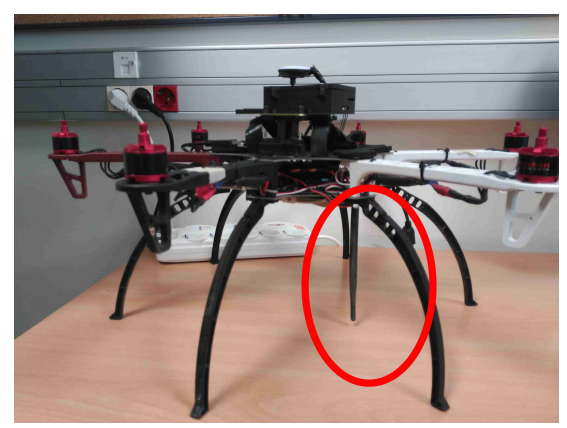

(b) Vertical

Figure 9: View of the antenna orientation.

graphic information (latitude, longitude, altitude). Since the receiver records the sender's geographical location, the distance between the drone and the moving vehicle can be directly inferred by comparing the sender's location and the receiver's location.

The packet delivery ratio is calculated by comparing the number of packets sent at the sender side and the number of packets received for a specific time interval. For our study, we have in fact analyzed both endpoints, sender, and receiver, as both contain a log file that is stored in each device. The log files are then analyzed to compare and calculate the ratio of packets sent and received by considering the geographical information inside.

In order to measure the potential factors that could affect the communi- 


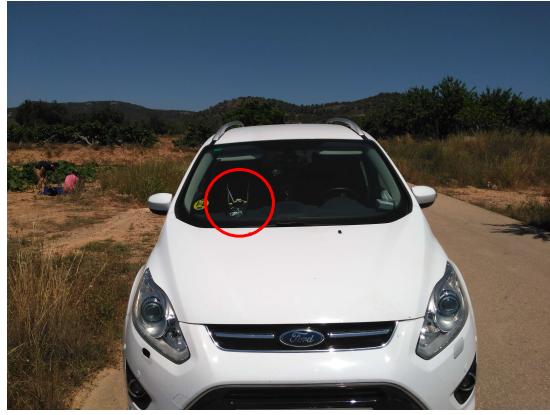

(a) Inside the Vehicle.

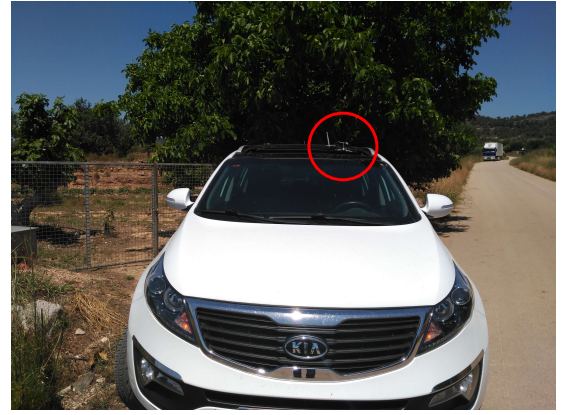

(b) Outside the Vehicle.

Figure 10: Views of the antenna location.

cation's performance, we have selected three variables for the experiment: the altitude of the sender (the drone), the sender's antenna orientation, and finally the receiver's antenna location. First, the drone's altitude will have a clear effect to the LOS probability, as the higher the drone is, the lesser the probability of finding obstacles, and so we considered two altitudes: 40m and $100 \mathrm{~m}$, respectively. The orientation of the drone antenna also affects the communications range, as the antenna waves propagate according to a different radiation pattern. So, we considered horizontal and vertical orientations (see Figure 9). Finally, testing with different antenna positions in the vehicle is also interesting to study as the communication will suffer degradation due to the vehicle's metal elements (see Figure 10. So at the vehicle side, we tested two different positions for the antenna: inside and outside (rooftop).

In order to simplify the definition of our scenarios, we have categorized them (see Table 1) depending on the UAV's altitude, antenna position at the car, and antenna orientation at the UAV. The naming defined in Table 1 will be used to indicate each scenario in the sections that follow.

\section{Experimental Results}

The experimental results are presented in heatmaps (see Figures 11 and 12 . for different drone altitudes. Each point represents locations where groups of 
Table 1: Scenario Categories

\begin{tabular}{|r|r|r|r|}
\hline Scenario & UAV Altitude & Car's Ant. Position & UAV's Ant. Orientation \\
\hline \hline Scenario 1 & $100 \mathrm{~m}$ & Outside & Vertical \\
\hline Scenario 2 & $100 \mathrm{~m}$ & Inside & Vertical \\
\hline Scenario 3 & $100 \mathrm{~m}$ & Outside & Horizontal \\
\hline Scenario 4 & $100 \mathrm{~m}$ & Inside & Verizontal \\
\hline Scenario 5 & $40 \mathrm{~m}$ & Outside & Vertical \\
\hline Scenario 6 & $40 \mathrm{~m}$ & Inside & Horizontal \\
\hline Scenario 7 & $40 \mathrm{~m}$ & Outside & Horizontal \\
\hline Scenario 8 & $40 \mathrm{~m}$ & Inside &
\end{tabular}

packets were successfully received. The points have different colors according to the associated delivery ratio. In terms of the relationship between communications range and packet delivery ratio, the results obtained are depicted in Figure 13. These results will then be modeled in the following section.

\section{1. $100 m$ altitude}

The results of the experiments made with the drone located at the higher altitude are shown in figure11. The best results are achieved by putting the car's antenna outside and having the drone's antenna pointing down vertically (Scenario 1). We can see in figure 11a that, in this situation, the communication reaches up to 3 kilometers. When the highest distance is reached, the delivery ratio is roughly above $10 \%$. Up until $1.4 \mathrm{~km}$, the delivery ratio is above $90 \%$. At $2.8 \mathrm{~km}$ range, we can see that this ratio falls from about 0.7 to less than 0.5. As expected, the furthest the car is, the fewer packets are received.

On the other hand, if we put the receiver's antenna inside the car (Scenario 2 ), the results are slightly worse. As we can see in Figure 11b, the reception trend has the same pattern as in the previous scenario, although the delivery ratio is slightly reduced. This is due to the presence of the car's materials, especially metals, which block the signal. At $2.8 \mathrm{~km}$ range, the delivery ratio is 


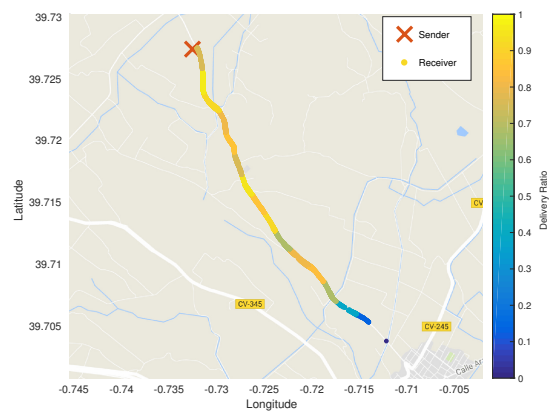

(a) Scenario 1: Outside, Vertical.

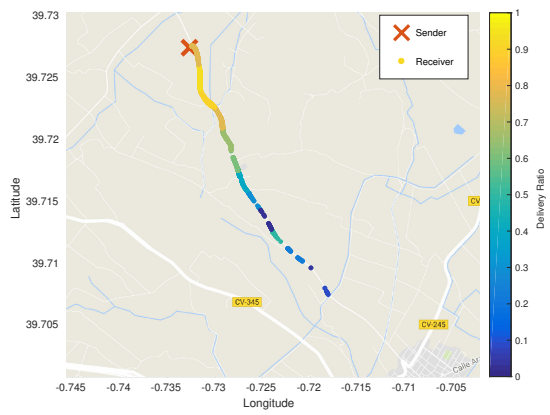

(c) Scenario 3: Outside, Horizontal.

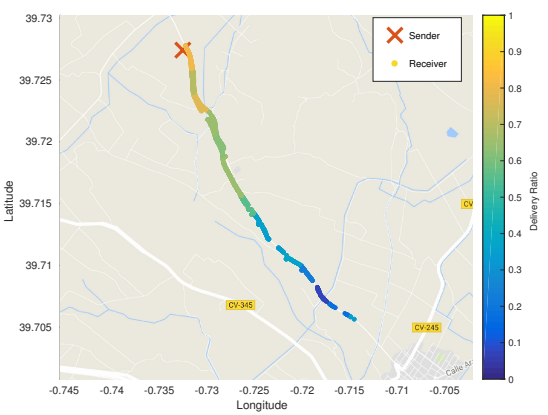

(b) Scenario 2: Inside, Vertical.

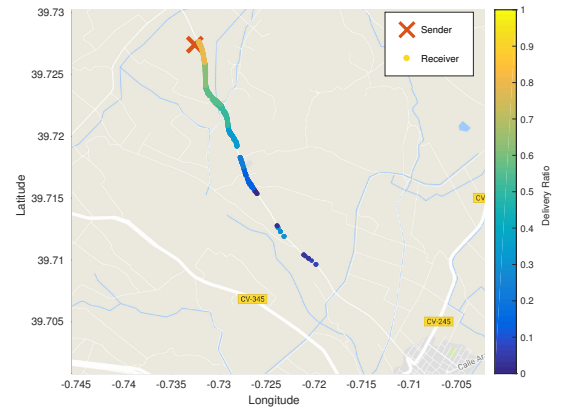

(d) Scenario 4: Inside, Horizontal.

Figure 11: Heat Maps for the different scenarios where the drone's altitude is at 100 meters.

Each plot shows the packet delivery ratio depending on the sender's antenna orientation (Vertical, Horizontal), and the receiver's antenna location (Inside, outside). 
found to be about $20 \%$. On the other hand, at the furthest point of reception, variables as in the previous scenario. Figure 12 shows the new results achieved. In general, we can see that the range is reduced when compared to the results achieved at 100 meters. We can also observe the impact of a little hill between the road that is located at coordinates latitude 39.719058, and longitude 370 -0.729005 , thus reducing drastically the delivery rate.

As in the previous experiments, we find that scenario 5 achieves the best results in this group, being that the communications range reaches up to 1.5 kilometers. At $1 \mathrm{~km}$, though, the packet delivery ratio already starts to drop to less than $20 \%$.

375

When we locate the antenna inside the vehicle (Scenario 6), the result is less satisfactory than the previous one. By having a lower range, the delivery ratio is lower for all distances when compared to the previous scenario. Another interesting fact is the ratio produced for the first few meters. In the case of 


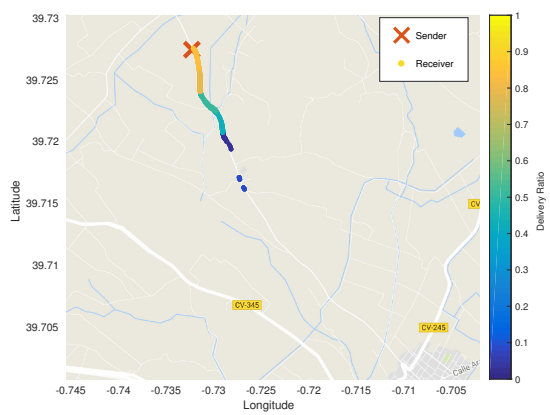

(a) Scenario 5: Outside, Vertical.

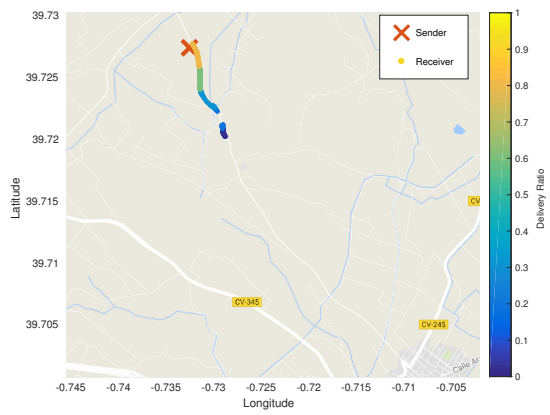

(c) Scenario 7: Outside, Horizontal.

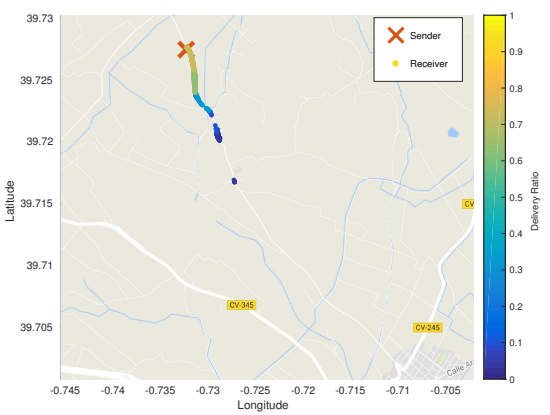

(b) Scenario 6: Inside, Vertical.

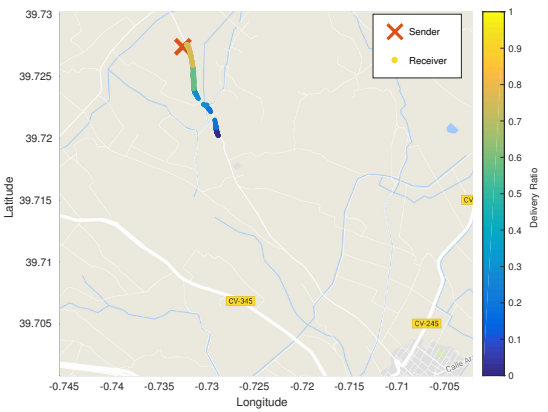

(d) Scenario 8: Inside, Horizontal.

Figure 12: Heat Maps for the different scenarios where the drone's altitude is of 40 meters.

Each plot shows the packet delivery ratio depending on the sender's antenna orientation (Vertical, Horizontal) and the receiver's antenna location (inside, outside). 
scenario 6 , this ratio is not decreasing in the range from 0 to 250 meters. In

This is due to the orientation of the antenna at the drone, as having the antenna pointing directly to the receiver would represent worst-case conditions in terms of energy radiation from that antenna. The maximum distance reached in this scenario is $1.3 \mathrm{~km}$.

385 the results are more standard, meaning that, as the distance increases, the delivery ratio attenuates in a monotonous manner. However, in terms of range and delivery ratio values, having the vehicle's antenna outside (scenario 7) still achieves lower performance than for the two previous scenarios (scenarios 5 and is of $1.3 \mathrm{~km}$, the maximum distance covered in this scenario is of less than 1 $\mathrm{km}$. At 500 meters, the packet delivery ratio has already dropped to $43 \%$.

Putting the antenna inside the vehicle while having the drone's antenna pointing horizontally is, as expected, the worst possible combination of all,

\section{Communications Modeling}

Using the results of the previous experiments, we now proceed by modeling communications based on the different factors being studied (drone's altitude, transmitter antenna orientation, and receiver antenna location). Notice that our model was obtained based on the number of packets received at each registered position. As the packet delivery ratio is calculated for a small distance interval, we have performed curve fitting to derive the optimal parameters. The models generated can then be integrated into simulation tools to have more realistic 


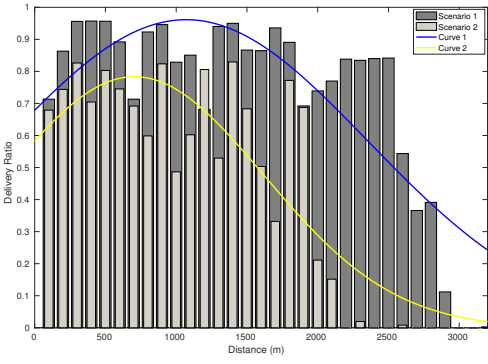

(a) Delivery ratio at Scenario 1 and 2 .

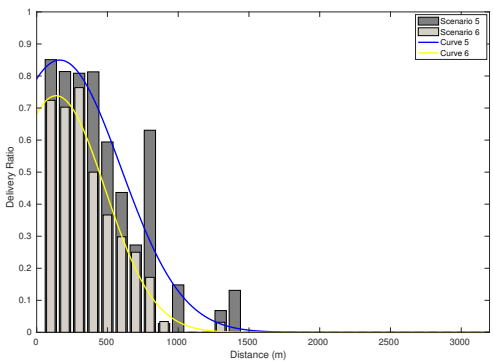

(c) Delivery ratio at Scenario 5 and 6 .

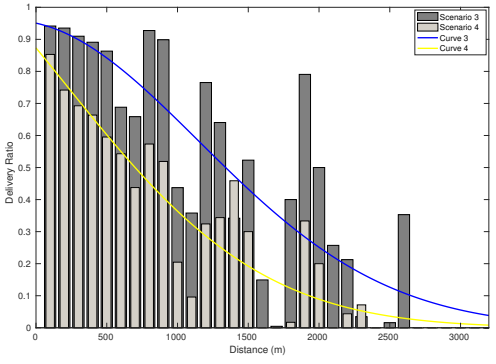

(b) Delivery ratio at Scenario 3 and 4 .

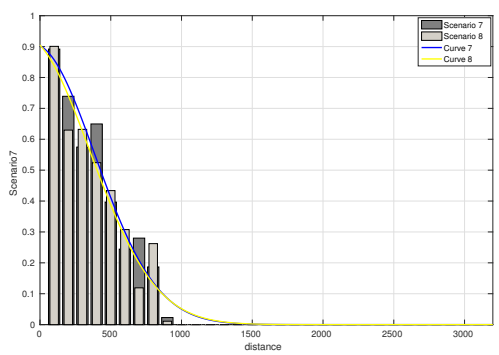

(d) Delivery ratio at Scenario 7 and 8 .

Figure 13: Curve Fittings of Delivery Ratio vs Distance, with the antenna put Inside or Outside the vehicle

performance results.

Specifically, for modeling this system, we used the location of the received packets (latitudes, longitudes, and altitudes), and their corresponding timestamp. The sender also stores information about how many packets were sent and when which can then be compared to the packets received to calculate the delivery ratio based on distance ranges. We have sliced the distance range into intervals of 100 meters. The delivery ratio is then calculated for each interval of distance range. The delivery ratio information for all scenarios tested is then plotted on a bar chart. Using this information, we continue by performing curve fitting using the nonlinear least-square Marquardt-Levenberg algorithm. This curve fitting allows deriving a general model for the packet delivery ratio vs. distance under different conditions.

The curve fitting results are presented in Figure 13 We have tried to find 
a common model that would suit our variables (antenna orientation, antenna location, and UAV altitude). The goal is having fitting parameters that can vary from one scenario to another while maintaining the same function. Thus, the selected fitting function was a modified Gaussian, and the best fitting was evaluated for the different cases studied:

$$
f(x)=a \cdot e^{-\frac{(x-b)^{2}}{2 c^{2}}}
$$

Based on equation 1, we have three parameters for each scenario: $a, b$, and c. These parameters take the values shown in Table 2. We have also added the $R^{2}$ value to represent the goodness-of-fit. Based on the table mentioned, for each scenario, the model explains all the variability of the response data around its mean. The parameter is represented as R-squared, which is a statistical measure of how close the data are to the fitted regression line. In our fitting of the delivery ratio and distance values obtained from the experiment, it shows a high value of R-squared, in which the value ranges from 0.7162 to 0.9844 . Hence, the model represents an adequate fit.

As depicted in Figure 13, the curve that represents those scenarios where the antenna is placed outside the vehicle has higher values than the curve representing those scenarios where the antenna is located inside the vehicle.

Comparing the curve that represents the scenarios that involve pointing down the antenna (vertical) to the opposite case (horizontal orientation), we 440 can see that some curves bend, while others do not. The curves that bend are the curves that represent a scenario having the antenna pointing down (vertical). This is expectable as, when the car is just below the drone's position, the delivery ratio becomes lower, increasing when the car gets a bit farther from the drone. This phenomenon is due to the fact that the antenna installed on the UAV is 445 an omnidirectional antenna, thereby radiating power uniformly in one plane, with the power decreasing with the elevation angle above or below that plane until it theoretically reaches zero on the antenna's axis [43. Hence, the signal that propagates from the antenna has its strongest power when the receiver is 
Table 2: Value of parameters $a, b$ and $c$ for each scenario with its $R^{2}$ value .

\begin{tabular}{|l|r|r|r|r|}
\cline { 2 - 5 } \multicolumn{1}{c|}{} & \multicolumn{1}{c|}{$a$} & \multicolumn{1}{c|}{$b$} & \multicolumn{1}{c|}{$c$} & $R^{2}$ \\
\hline Scenario 1 (Curve 1) & 0.9613 & 1071 & 1815 & 0.7162 \\
\hline Scenario 2 (Curve 2) & 0.7834 & 705.4 & 1296 & 0.8132 \\
\hline Scenario 3 (Curve 3) & 0.9593 & -180.2 & 1883 & 0.7646 \\
\hline Scenario 4 (Curve 4) & 1.24 & -11.49 & 1941 & 0.8627 \\
\hline Scenario 5 (Curve 5) & 0.8497 & 163.4 & 609.5 & 0.9279 \\
\hline Scenario 6 (Curve 6) & 0.7385 & 137.7 & 488.9 & 0.9844 \\
\hline Scenario 7 (Curve 7) & 0.9083 & -48.59 & 616.4 & 0.9786 \\
\hline Scenario 8 (Curve 8) & 0.932 & -113.1 & 654.2 & 0.971 \\
\hline
\end{tabular}

perpendicular to it. On the other hand, when the receiver is located parallel to the antenna (in our case, just below), it will receive a weaker signal.

From another perspective, when comparing the curves for the scenarios where we vary the drone's altitude, it quickly becomes clear that higher altitudes are associated to greater distances. For Scenarios 1 and 2, the curves continue up to distances of more than 3.2 kilometers. For Scenario 3, the curve stops at $3 \mathrm{~km}$ and reaches 200 additional meters on Scenario 4. On the other hand, for both Scenarios 5 and 6, the resulting fitting function cannot reach more than $1.5 \mathrm{~km}$. On the last two scenarios, the maximum point reached by the resulting curve is $1.3 \mathrm{~km}$. That is, for the scenarios when the drone's altitude is of $40 \mathrm{~m}$, these cut-off values precisely correspond to locations where LOS is blocked by hills, as shown in figure $6 \mathrm{~b}$.

It is worth pointing out that, since our experiments were located in a quite remote rural area, no significant interference was present that could hinder the communications band. Thus, the results achieved are quite reasonable as, for this situation, the fitting suits an AWGN (Additive White Gaussian Noise) channel model, meaning that only the effect of additive white Gaussian noise is noticeable. Also, we find that the only obstacles that hindered the communication were little hills that blocked line of sight in some areas of the selected 
scenarios.

\section{7. =Model Applicability and Comparison Against Existing Models}

In this section we discuss the novelty and applicability of our model by comparing it against other existing propagation models, and explaining how it could be used as a part of existing network simulators.

\subsection{Comparison with Existing Models}

The characterization of a data link between two endpoints requires studying the signal propagation conditions in the target environment. The line of sight conditions and the presence of obstacles clearly affect the channel model due to the signal reflections and Doppler spread effects. This is specially true for vehicular communications, where it can be affected by the presence of buildings, terrain profile, or the car metal structure itself.

480

Various empirical path loss models have been proposed to address the signal propagation phenomenon. However, very specific transmission conditions cannot be addressed by these models, as they only provide a generic propagation behavior. Regarding vehicular communication settings, we have found in previous works that different types of intersections have their own characteristics that define the communications performance when the visibility between two nodes is obstructed by either buildings or trees [44]. In our current work, we have found that terrain conditions (e.g hills, mountains) can be a critical factor affecting communication between UAVs and ground vehicles.

To clearly demonstrate the usefulness and novelty of the models derived from this work, we have also compared our results with those obtained from simulation experiments that mimic the real-life experimental settings. We have chosen the $\mathrm{OMNeT}++[45$ tool for simulation tests. Table 3 presents the simulation parameters adopted. Notice that, in order to get simulation results comparable to the real experimental results, we have set the simulation parameters in accordance to real-life settings, except for the Path Loss Model, which is the parameter under analysis. 
Table 3: Simulation Parameters

\begin{tabular}{|r|r|}
\hline Parameters & Values \\
\hline Transmitter Power & $200 \mathrm{~mW}$ \\
\hline Antenna Gain & $5 \mathrm{~dB}$ \\
\hline Packet Size & 1400 Bytes \\
\hline Packet Rate & $10 \mathrm{~Hz}$ \\
\hline Transmitter's Altitude & $40 \mathrm{~m} / 100 \mathrm{~m}$ \\
\hline Path Loss Model & Free-Space, Rayleigh Fading \\
\hline
\end{tabular}

For our simulation experiments we considered two existing empirical path loss models. The first one was the Free-space path loss model [46], and the second one was the Rayleigh fading [47 model. Simulation experiments include two nodes, one static and another moving, thereby resembling the drone and car of the real experiment. Then, we have measured the packets sent from the transmitter, and those received correctly at the receiver node. Afterward, we have calculated the packet delivery ratio progressively, depending on the distance between the two nodes. This way, we obtained a graph for packet delivery ratio versus distance, the same way as we did with the results obtained in the real experiment.

Figure 14 combines the results for the real tests and the simulation tests for comparison. The curves have distinct colors, where Scenario 1 represents the real experiment with the drone flying at 100 meters in a green curve, and Scenario 5 represents the real experiment with the drone flying at 40 meters in a red curve. In both cases, these results correspond to the best case scenario, with the UAV antenna pointing down, and the vehicle's antenna located on top of it. Regarding the Rayleigh results represented in the figure in a blue curve, these correspond to the simulation test using the Rayleigh fading model, while the Free-Space line (in cyan color) corresponds to simulation results using the FreeSpace path loss model. Regarding the altitude parameter, simulation results actually show that the impact of varying the UAV's altitude is negligible, being 


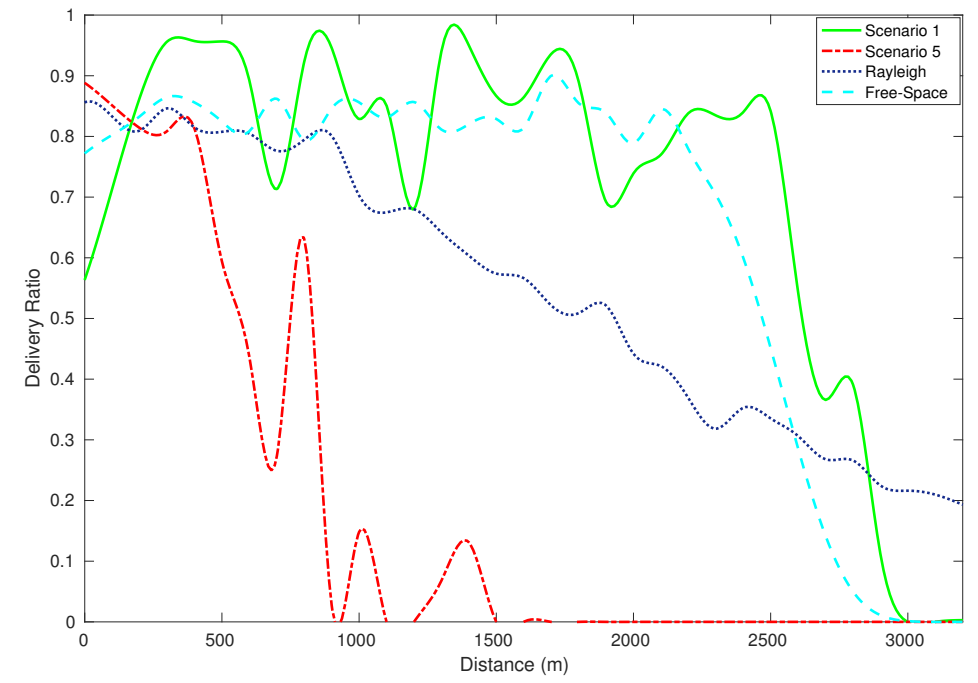

Figure 14: Comparison of results obtained from simulations and real experiments.

that very similar results are obtained when having the transmitter at 40 meters or 100 meters. Hence, the curves that are shown in the figure are applicable for the channel model regardless of the transmitter's altitude chosen.

Based on the results presented in Figure 14, we can notice that the Rayleigh fading model does not represent the performance of the communications achieved when flying at either 40 or 100 meters. In fact, the Rayleigh fading model only shows a progressive decline as the distance between endpoints increases. In addition, even though in the real experiment the communication was lost after 3.2 kilometers, the curve still shows a successful packet delivery beyond that distance. Overall, we can conclude that it cannot represent the performance for UAV-to-car communication conditions.

Regarding the Free Space path loss model, it is more adequate for the scenario being tested. We can see that, as the distance increases, the power attenuates drastically after a certain distance. This is shown in its curve when it attains 2000 meters, being that the curve plunges from 0.85 to less than 0.5 for a distance variation of only 200 meters. This trend is similar to the curve 
that represents the real experiment when the transmitter was at an altitude of cause line-of-sight obstructions. However, compared to the curve that represents the experiments having the transmitter at 40 meters of altitude, we find that the Free Space model is no longer applicable. Now, since communication obstructions were present between the drone that acts as the transmitter and the moving vehicle, the packet delivery ratio drops for much shorter distances.

Taking into account the results presented above, we conclude that the existing channel models still cannot address the specific characteristics of UAV-toCar communication scenarios. In fact, we found that the free-space path loss model is applicable, but only when there is line of sight between both endpoints.

545 In case the terrain profile causes obstructions, then traditional models are no longer applicable, and an obstruction model specific to each particular scenario is required.

\subsection{Applicability of the proposed Model in Simulation}

Knowing that our proposed model can be an alternative for defining communications performance in UAV-to-car scenarios, our intended goal is to use this model for larger scale simulations. In order to adapt our model to simulation environments, information regarding the terrain profile in the target area should be known in advance. Hence a topographic map with elevation information would be needed for the simulation to be as realistic as possible.

By analyzing a 3D map (with elevation information), one can characterize the line-of-sight conditions between UAVs and cars by classifying the elevation and landforms as obstacles to communication. This classification process can be done before the actual simulation. Widely used map providers such as OpenStreetmap 48 include elevation information, which make the adoption of our model feasible. This way, the adoption of the models derived in this paper can be used in combination with line-of-sight availability based on information of the actual scenario topology, which is used to determine whether signal blocking due to obstacles will take place or not, prior to checking the packet delivery 
feasibility accounting for distance-related loss.

\section{Conclusions}

In this paper, we have studied the packet delivery effectiveness on UAV-tocar communications. For this study, we have varied the drone's altitude, the transmitter antenna orientation, and the receiver antenna location. According to our experiments, the best scenario for UAV to car communications takes place when the drone's antenna is pointing down (vertical), the car's antenna is located outside the vehicle, and the drone's altitude is very high (100 meters, near the maximum allowed limit according to the legislation in different countries), thereby helping to avoiding any LOS obstruction. Under this setting, the communications range achieved was of 3.2 kilometers.

In addition, based on the experimental results obtained, we have also found the best-fitting function and applied regression to provide a generic model applicable to the different situations under study. The model is based on the modified Gaussian function, which offers a suitable results representation such as the ones obtained in our tests. In fact, by merely adjusting two values of the fitting function, we can adapt it to the different scenarios tested. Simulation results have also shown that the derived models keep some relationship with the standard Free Space model when LOS conditions are met, but not otherwise.

As future work, we will study in more detail the impact of obstructions present at different altitudes. In particular, the presence of hills as an obstruction to the line of sight communications is an important factor to take into account, and thus integrating 3D maps in network simulation tools can be useful to improve the degree of realism of our experiments.

\section{Acknowledgment}

This work was partially supported by the "Ministerio de Economía y Competividad, Programa Estatal de Investigación, Desarollo e Innovación Orien- 
tada a los Retos de la Sociedad, Proyectos I+D+I 2014", Spain, under Grants TEC2014-52690-R and BES-2015-075988.

\section{References}

[1] K. Sjoberg, P. Andres, T. Buburuzan, A. Brakemeier, Cooperative intelligent transport systems in europe: Current deployment status and outlook, IEEE Vehicular Technology Magazine 12 (2) (2017) 89-97.

[2] F. J. Martinez, C.-K. Toh, J.-C. Cano, C. T. Calafate, P. Manzoni, Emergency services in future intelligent transportation systems based on vehicular communication networks, IEEE Intelligent Transportation Systems Magazine 2 (2) (2010) 6-20.

[3] S. A. Hadiwardoyo, S. Patra, C. T. Calafate, J.-C. Cano, P. Manzoni, An intelligent transportation system application for smartphones based on vehicle position advertising and route sharing in vehicular ad-hoc networks, Journal of Computer Science and Technology 33 (2) (2018) 1-14, (in press).

605

[6] M. Khabazian, S. Aïssa, M. Mehmet-Ali, Performance modeling of safety messages broadcast in vehicular ad hoc networks, IEEE transactions on intelligent transportation systems 14 (1) (2013) 380-387.

[7] E. Hossain, G. Chow, V. C. Leung, R. D. McLeod, J. Mišić, V. W. Wong, survey, Computer Communications 33 (7) (2010) 775-793. 
[8] E. Ndashimye, S. K. Ray, N. I. Sarkar, J. A. Gutiérrez, Vehicle-toinfrastructure communication over multi-tier heterogeneous networks: A survey, Computer Networks 112 (2017) 144-166.

[9] S. Waharte, N. Trigoni, Supporting search and rescue operations with uavs, in: Emerging Security Technologies (EST), 2010 International Conference on, IEEE, 2010, pp. 142-147.

[10] C. Yuan, Y. Zhang, Z. Liu, A survey on technologies for automatic forest fire monitoring, detection, and fighting using unmanned aerial vehicles and remote sensing techniques, Canadian journal of forest research 45 (7) (2015) 783-792.

[11] I. Bekmezci, O. K. Sahingoz, Ş. Temel, Flying ad-hoc networks (fanets): A survey, Ad Hoc Networks 11 (3) (2013) 1254-1270.

[12] I. Jawhar, N. Mohamed, J. Al-Jaroodi, D. P. Agrawal, S. Zhang, Communication and networking of uav-based systems: Classification and associated architectures, Journal of Network and Computer Applications 84 (2017) 93-108.

[13] A. Bujari, C. T. Calafate, J.-C. Cano, P. Manzoni, C. E. Palazzi, D. Ronzani, Flying ad-hoc network application scenarios and mobility models, International Journal of Distributed Sensor Networks 13 (10) (2017) 1550147717738192 .

[14] L. Chaimowicz, A. Cowley, D. Gomez-Ibanez, B. Grocholsky, M. Hsieh, H. Hsu, J. Keller, V. Kumar, R. Swaminathan, C. Taylor, Deploying airground multi-robot teams in urban environments, in: Multi-Robot Systems. From Swarms to Intelligent Automata Volume III, Springer, 2005, pp. 223-234.

[15] H. Menouar, I. Guvenc, K. Akkaya, A. S. Uluagac, A. Kadri, A. Tuncer, Uav-enabled intelligent transportation systems for the smart city: Applications and challenges, IEEE Communications Magazine 55 (3) (2017) 22-28. 
[16] E. Yanmaz, M. Quaritsch, S. Yahyanejad, B. Rinner, H. Hellwagner, C. Bettstetter, Communication and coordination for drone networks, in: Ad Hoc Networks, Springer, 2017, pp. 79-91.

[17] K. Daniel, B. Dusza, A. Lewandowski, C. Wietfeld, Airshield: A system-ofsystems muav remote sensing architecture for disaster response, in: Systems conference, 2009 3rd Annual IEEE, IEEE, 2009, pp. 196-200.

[18] W. Zafar, B. M. Khan, Flying ad-hoc networks: technological and social implications, IEEE Technology and Society Magazine 35 (2) (2016) 67-74.

[19] J. Wang, C. Jiang, Z. Han, Y. Ren, R. G. Maunder, L. Hanzo, Taking drones to the next level: Cooperative distributed unmanned-aerialvehicular networks for small and mini drones, Ieee vehIcular technology magazIne 12 (3) (2017) 73-82.

[20] M. B. Yassein, N. A. Damer, Flying ad-hoc networks: Routing protocols, mobility models, issues, International Journal Of Advanced Computer Science And Applications 7 (6) (2016) 162-168.

[21] A. Al-Hourani, S. Kandeepan, A. Jamalipour, Modeling air-to-ground path loss for low altitude platforms in urban environments, in: Global Communications Conference (GLOBECOM), 2014 IEEE, IEEE, 2014, pp. 28982904.

[22] Y. Zhou, N. Cheng, N. Lu, X. S. Shen, Multi-uav-aided networks: aerialground cooperative vehicular networking architecture, ieee vehicular technology magazine 10 (4) (2015) 36-44.

[23] S. Jia, L. Zhang, Modelling unmanned aerial vehicles base station in ground-to-air cooperative networks, IET Communications 11 (8) (2017) 1187-1194.

[24] O. S. Oubbati, A. Lakas, F. Zhou, M. Güneş, N. Lagraa, M. B. Yagoubi, Intelligent uav-assisted routing protocol for urban vanets, Computer Communications 107 (2017) 93-111. 
[25] M. Asadpour, B. Van den Bergh, D. Giustiniano, K. Hummel, S. Pollin, B. Plattner, Micro aerial vehicle networks: An experimental analysis of challenges and opportunities, IEEE Communications Magazine 52 (7) (2014) 141-149.

[26] S. Rohde, C. Wietfeld, Interference aware positioning of aerial relays for cell overload and outage compensation, in: Vehicular Technology Conference (VTC Fall), 2012 IEEE, IEEE, 2012, pp. 1-5.

[27] G. Tuna, B. Nefzi, G. Conte, Unmanned aerial vehicle-aided communications system for disaster recovery, Journal of Network and Computer Applications 41 (2014) 27-36.

[28] J. Q. Cui, S. K. Phang, K. Z. Ang, F. Wang, X. Dong, Y. Ke, S. Lai, K. Li, $\mathrm{X}$. Li, J. Lin, et al., Search and rescue using multiple drones in post-disaster situation, Unmanned Systems 4 (01) (2016) 83-96.

[29] M. İ. Akbaş, G. Solmaz, D. Turgut, Molecular geometry inspired positioning for aerial networks, Computer Networks 98 (2016) 72-88.

[30] L. T. Lilien, L. Ben Othmane, P. Angin, A. DeCarlo, R. M. Salih, B. Bhargava, A simulation study of ad hoc networking of uavs with opportunistic resource utilization networks, Journal of Network and Computer Applications 38 (2014) $3-15$.

[31] M. Erdelj, M. Król, E. Natalizio, Wireless sensor networks and multi-uav systems for natural disaster management, Computer Networks 124 (2017) $72-86$.

[32] P. Shilin, R. Kirichek, A. Paramonov, A. Koucheryavy, Connectivity of vanet segments using uavs, in: International Conference on Next Generation Wired/Wireless Networking, Springer, 2016, pp. 492-500.

[33] W. Fawaz, R. Atallah, C. Assi, M. Khabbaz, Unmanned aerial vehicles as store-carry-forward nodes for vehicular networks, IEEE Access 5 (2017) 23710-23718. 
[34] X. Wang, L. Fu, Y. Zhang, X. Gan, X. Wang, Vdnet: an infrastructure-less uav-assisted sparse vanet system with vehicle location prediction, Wireless Communications and Mobile Computing 16 (17) (2016) 2991-3003.

[35] V. Sharma, R. Kumar, G-fanet: an ambient network formation between ground and flying ad hoc networks, Telecommunication Systems 65 (1) (2017) 31-54.

[36] E. Yanmaz, R. Kuschnig, C. Bettstetter, Achieving air-ground communications in 802.11 networks with three-dimensional aerial mobility, in: INFOCOM, 2013 Proceedings IEEE, IEEE, 2013, pp. 120-124.

[37] T. Andre, K. A. Hummel, A. P. Schoellig, E. Yanmaz, M. Asadpour, C. Bettstetter, P. Grippa, H. Hellwagner, S. Sand, S. Zhang, Applicationdriven design of aerial communication networks, IEEE Communications Magazine 52 (5) (2014) 129-137.

[38] F. Fabra, C. T. Calafate, J.-C. Cano, P. Manzoni, On the impact of interuav communications interference in the $2.4 \mathrm{ghz}$ band, in: Wireless Communications and Mobile Computing Conference (IWCMC), 2017 13th International, IEEE, 2017, pp. 945-950.

[39] E. A. Marconato, J. A. Maxa, D. F. Pigatto, A. S. Pinto, N. Larrieu, K. R. C. Branco, Ieee 802.11 n vs. ieee 802.15. 4: a study on communication qos to provide safe fanets, in: Dependable Systems and Networks Workshop, 2016 46th Annual IEEE/IFIP International Conference on, IEEE, 2016, pp. 184-191.

[40] S. Temel, I. Bekmezci, Lodmac: Location oriented directional mac protocol for fanets, Computer Networks 83 (2015) 76-84.

${ }_{725}$ [41] S. M. Tornell, S. Patra, C. T. Calafate, J.-C. Cano, P. Manzoni, Grcbox: extending smartphone connectivity in vehicular networks, International Journal of Distributed Sensor Networks 11 (3) (2015) 478064. 
[42] ETSI, Intelligent transport systems (its); vehicular communications; basic set of applications; part 3: Specification of decentralized environmental notification basic service, TS 102 637-3, ETSI (2010).

[43] N. McDonald, Omnidirectional pattern directivity in the presence of minor lobes: Revisited, IEEE Transactions on Antennas and Propagation Magazine 41 (2) (1999) 63-68.

[44] S. A. Hadiwardoyo, A. Tomás, E. Hernández-Orallo, C. T. Calafate, J.-C. Cano, P. Manzoni, Empirical study and modeling of vehicular communications at intersections in the 5 ghz band, Mobile Information Systems 2017.

[45] A. Varga, R. Hornig, An overview of the omnet++ simulation environment, in: Proceedings of the 1st international conference on Simulation tools and techniques for communications, networks and systems \& workshops, ICST (Institute for Computer Sciences, Social-Informatics and Telecommunications Engineering), 2008, p. 60.

[46] H. T. Friis, A note on a simple transmission formula, Proceedings of the IRE 34 (5) (1946) 254-256.

[47] B. Sklar, Rayleigh fading channels in mobile digital communication systems. i. characterization, IEEE Communications magazine 35 (9) (1997) $136-146$.

[48] M. Haklay, P. Weber, Openstreetmap: User-generated street maps, IEEE Pervasive Computing 7 (4) (2008) 12-18. 